\title{
« Nous, les fauteuils... ». Essai sur le passage de la culture « debout » à celle du « fauteuil roulant »
}

\section{Anne Marcellini}

\section{(2) OpenEdition}

\section{Journals}

Édition électronique

URL : http://journals.openedition.org/trema/2445

DOI : $10.4000 /$ trema.2445

ISSN : 2107-0997

Éditeur

Faculté d'Éducation de l'université de Montpellier

Édition imprimée

Date de publication : 24 octobre 1997

Pagination : $8-21$

ISSN : 1167-315X

Référence électronique

Anne Marcellini, « « Nous, les fauteuils... ». Essai sur le passage de la culture « debout » à celle du « fauteuil roulant » », Tréma [En ligne], Hors série N¹ | 1997, mis en ligne le 28 septembre 2015, consulté le 19 avril 2019. URL : http://journals.openedition.org/trema/2445; DOI : 10.4000/ trema.2445

Ce document a été généré automatiquement le 19 avril 2019

Trema 


\title{
« Nous, les fauteuils... ». Essai sur le passage de la culture « debout » à celle $\mathrm{du}$ « fauteuil roulant »
}

\author{
Anne Marcellini
}

1 «La culture est une nuit incertaine où dorment les révolutions d'hier, invisibles, repliées dans les pratiques, mais des lucioles et quelquefois de grands oiseaux nocturnes, la traversent, surgissements et créations qui tracent la chance d'un autre jour. Cette nuit océanique me fascine et m'interroge ». Michel De Certeau

2 Comment peut-on enseigner une « culture corporelle»? Pour tenter de donner une réponse à cette interrogation qui contient une notion controversée et floue, j'ai choisi de proposer ici un regard ethnographique sur un groupe social un peu particulier : les personnes en fauteuil roulant.

3 En effet, si ce groupe, en tant que sous-groupe de notre société, participe bien de la culture qui nous est commune à tous, la stigmatisation dont il fait l'objet, sa position de relative marginalité, et ses spécificités corporelles en font un groupe dans lequel la question de la «culture corporelle» mérite une attention certaine. En outre, un certain nombre de personnes en fauteuil roulant sont des personnes qui ont subi une atteinte traumatique. Elles ont donc été éduqués à vivre "debout", pour devoir ensuite apprendre, après un accident, à vivre "en fauteuil ». C'est la question du passage d'une situation à une autre que je voudrais traiter ici en tentant de répondre aux questions suivantes : Quels sont les usages du corps spécifiques repérables dans le groupe des personnes en fauteuil roulant? Existe-t-il des mécanismes ou des procédures de transmission de ceux-ci à l'intérieur de ce groupe ? Et si oui, lesquels? Je vous propose donc un regard sur ce groupe spécifique, pour tenter d'éclairer la question générale de « l'enseignement d'une culture corporelle ». 


\section{De l'atteinte du corps à de nouveaux usages du corps?}

4 Les atteintes corporelles majeures, telles que les paraplégies ou les tétraplégies d'origine traumatique ont été étudiées du point de vue de leurs conséquences psychologiques. La métamorphose corporelle engendrée par ces atteintes génère une déstructuration de l'image du corps et de l'image de soi. Elle oblige à une réorganisation sensori-motrice, psychologique et sociale au cours de laquelle les représentations du corps et de soi subissent une véritable révolution (Robert Murphy, 1987).

5 Au-delà de cette réorganisation psychologique, l'augmentation du nombre des personnes touchées et leur autonomisation progressive semble aboutir aujourd'hui à l'émergence d'un véritable groupe social. Ce groupe est extérieurement repérable par l'existence d'associations de personnes handicapées, de groupes de pressions et de groupes militants, de représentants politiques, d'une fédération sportive etc. Mais il est également subjectivement perçu, et un certain nombre de personnes handicapées catégorisent le social à partir de ce groupe d'appartenance : " Nous, les fauteuils... », " les handicapés mentaux c'est autre chose ", "vous, les valides». Lorsqu'on s'enquiert de l'identité de quelqu'un dont on parle, il s'agit de savoir si'l est «en fauteuil » ou «debout ». De nombreux indices soulignent le sentiment de l'entrée dans un autre groupe, voire dans un autre monde que constituent l'accident et l'obligation de vivre en fauteuil roulant : dans la présentation de soi, les sujets expliquent de quelle «cuvée » ils sont, où ils ont fait « leurs classes », pour situer dans le temps et dans l'espace leur entrée dans la vie « en fauteuil » et leur apprentissage de celle-ci.

A partir de ces constats de départ, nous voudrions montrer maintenant que ce groupe social présente aujourd'hui des usages du corps qui lui sont spécifiques et qui le différencient d'autres groupes.

\subsection{Usages sociaux du corps, et techniques du corps}

6 Claude Levi-Strauss rappelait, en 1950, dans son introduction à l'ouvrage de Marcel Mauss « Sociologie et anthropologie» que «Personne, en vérité, n'a encore abordé cette tâche immense dont Mauss soulignait l'urgente nécessité, à savoir l'inventaire et la description de tous les usages que les hommes, au cours de l'histoire et surtout à travers le monde, ont fait et continuent de faire de leur corps. "

Marcel Mauss, bien connu dans le champ de l'E.P.S., a développé l'expression de " techniques du corps" pour désigner «les façons dont les hommes, société par société, d'une façon traditionnelle, savent se servir de leur corps. » (1950, p. 365). Il inclut donc sous ce vocable aussi bien les techniques corporelles, que les attitudes corporelles. Il utilise également le terme d'habitus pour désigner la «manière d'être». La notion de « techniques de corps » au sens de Mauss renvoie donc à toutes les réalités groupales relatives à « l'art d'utiliser le corps humain».

Nous nous en tiendrons, pour cette première partie, à cette définition large, encore floue mais qui est fonctionnelle pour une première analyse purement descriptive qui vise ici à l'identification d'usages particuliers du corps. 


\subsection{Techniques du corps, attitudes et habitus corporel chez les personnes en fauteuil roulant}

7 Dans le cadre d'espaces de pratique sportive pour personnes handicapées physiques en particulier, mais aussi dans le cadre de la vie quotidienne, nous avons mené une étude de type ethnographique, par observation, visant dans un premier temps à repérer d'éventuels usages spécifiques du corps.

8 Dans cette perspective, nous nous sommes centrés sur le repérage de l'habitus corporel des personnes en fauteuil roulant, leur " manière d'être » corporelle, et sur l'identification des formes de présentation du corps, d'attitudes corporelles ou de techniques corporelles spécifiques.

9 Nous ne présenterons ici que quelques « modalités spécifiques d'usages du corps » que nous avons observées, en essayant de les catégoriser à partir de la grille proposée par Marcel Mauss. 


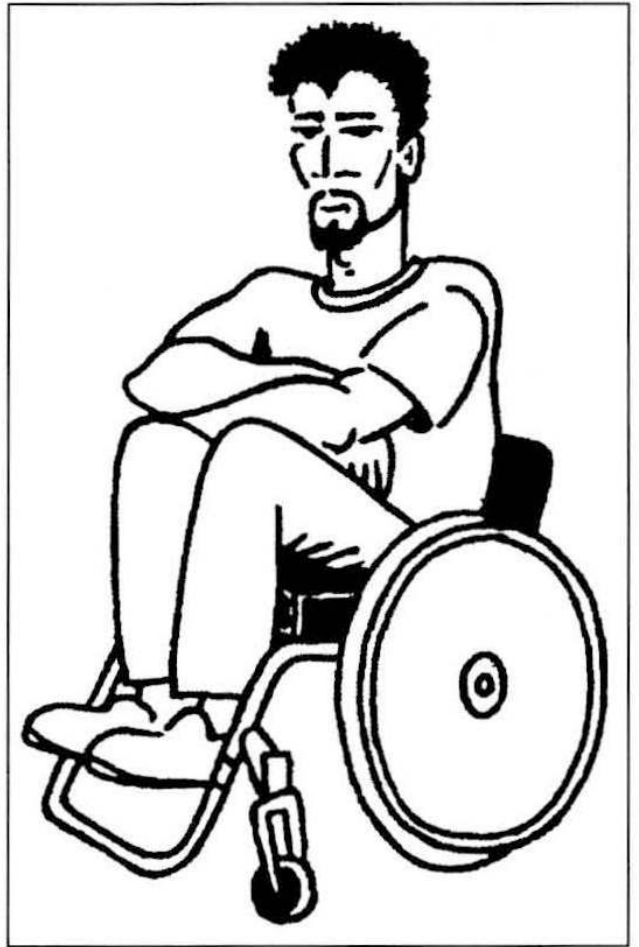

Les ATtitudes Corporelles AU Repos. Assis dans le fauteuil roulant avec les deux jambes sur la sangle. Dessin de Jean-Pierre Thérond.

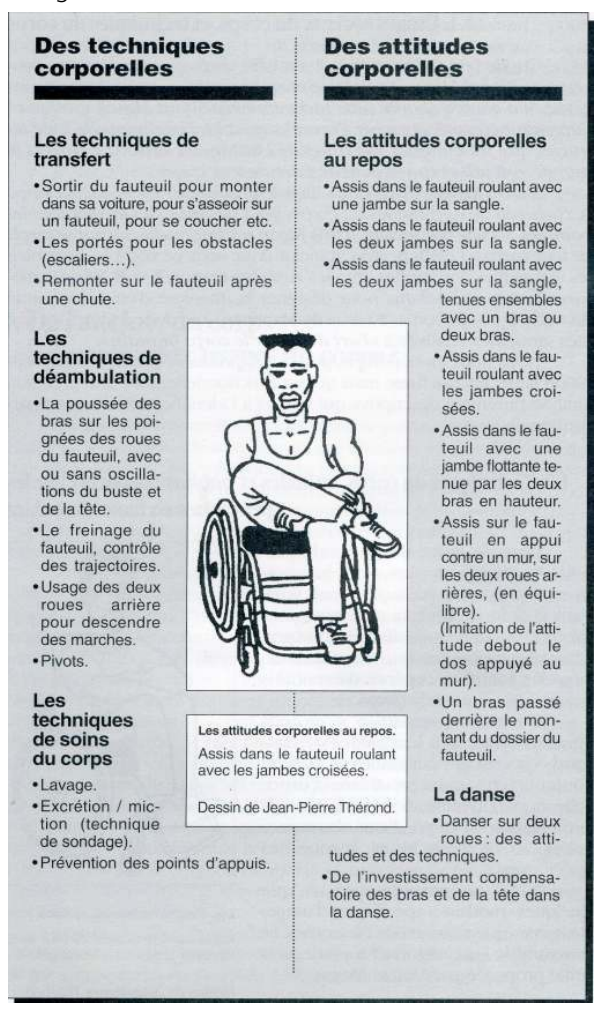




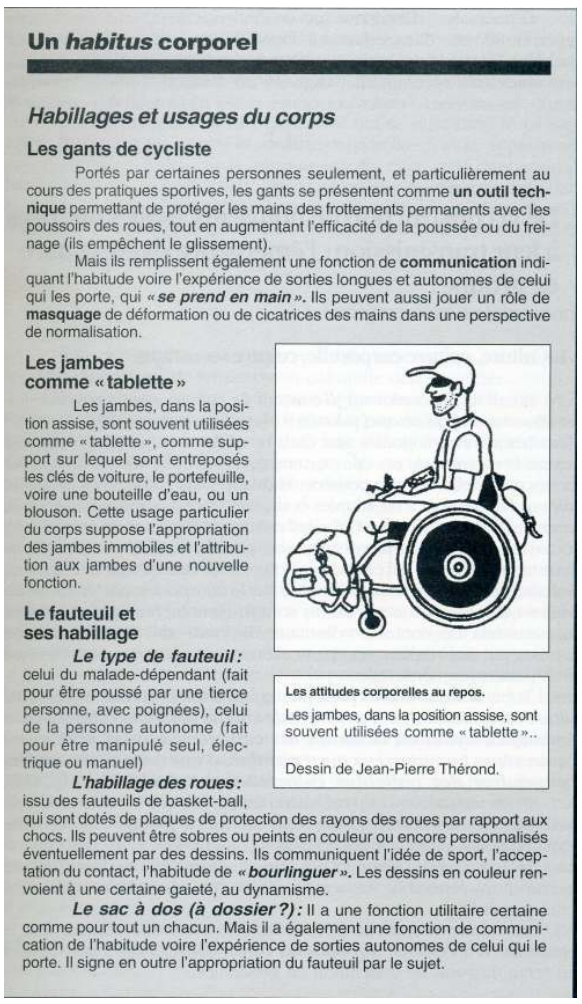

10 Cette analyse descriptive met en évidence des axes d'observations (façon de se tenir dans le fauteuil, façon de sortir du fauteuil, façon de s'habiller, façon de se déplacer etc.) qui permettent de différencier des personnes très récemment assignées au fauteuil roulant (les «nouveaux », les « novices ») et des personnes vivant en fauteuil (les « anciens »).

\section{De la construction de nouveaux usages du corps à leur transmission : l'émergence d'une culture de groupe?}

\subsection{Culture, culture corporelle, culture somatique}

11 Il faut ici s'accorder sur le concept de culture, pour essayer de tracer les contours de ce que peut-être une « culture corporelle ».

Les anthropologues voient dans la culture d'un peuple, sa manière de voir, l'ensemble de ses comportements types et de ses attitudes, et les choses matérielles qu'elle possède. (Edward Hall, 1959). Mais la culture ne peut être réduite à de simples coutumes dont on changerait comme on change de garde-robe. Dans les cultures différentes de la nôtre, on trouve une organisation sociale, une pensée et une conception de l'homme totalement différentes des nôtres. La culture agit directement, profondément et de manière durable sur le comportement, et les mécanismes qui relient l'une aux autres sont souvent inconscients, se situant donc au-delà du contrôle volontaire de l'individu. Cette dimension inconsciente de la culture sera particulièrement importante dans l'analyse de sa dimension corporelle.

Tobie Nathan (1994) présente, quant à lui, la culture ainsi : « Une culture est constituée par l'ensemble de tous les individus culturellement semblables, ayant en commun des 
caractères qui les distinguent d'autres êtres humains, eux aussi membres d'une culture, et capables d'engendrer des individus culturellement semblables» (p. 179).

Nous retiendrons ici particulièrement le fait que la culture, au-delà du «commun » observable qu'elle suppose dans le groupe culturel considéré, présente deux aspects qui nous intéressent plus particulièrement ici: tout d'abord elle contient une dimension inconsciente, ensuite le « commun » repérable ne serait culture que dans la mesure où il serait transmis.

La culture corporelle peut donc être entendue comme tout le « commun » d'un groupe à propos du corps, conscient et inconscient et qui serait transmis de génération en génération.

Si nous voulons parler de "culture corporelle», nous devons donc ici dépasser les seules «techniques du corps » au sens de Marcel Mauss pour aborder également tout ce qui a trait aux savoirs sur le corps, aux représentations de celui-ci, et plus globalement à « la manière de voir » le corps.

Cet élargissement de la perspective qui se situe dans le passage entre « techniques du corps » et «culture corporelle » sera ici appréhendé au travers de la question de la transmission. En effet, cette dimension de transmission de caractères spécifiques au groupe est déterminante dans la notion de culture: n'est culturel que ce qui est transmis à la génération suivante. Il va donc s'agir maintenant de savoir qu'est ce qui est transmis, et surtout comment cela est transmis.

\subsection{Enseignement ou Education?}

12 Nous pouvons nous appuyer sur différents auteurs pour repérer les différents modes de transmission culturelle déjà identifiés.

Marcel Mauss exprime clairement la dimension culturelle des techniques du corps en rappelant qu' "Il n'existe pas de façon <naturelle> de mouvoir son corps. ". Sans entrer dans une délimitation et une classification stricte, il souligne cependant que les techniques s'enseignent (exemple de la nage). Lorsqu'il parle ensuite des attitudes du corps en mouvement, comme par exemple la démarche qu'il classe comme «idiosyncrasie sociale », il explique que cela s'éduque. Il utilise ensuite le terme d' habitas du corps pour désigner la " manière d'être » avec le corps.

Il note ensuite que dans tous ces éléments de l'art d'utiliser le corps humain les faits d'éducation dominent. Il associe également cela à la notion d'imitation et parle d'imitation prestigieuse, dans le sens où l'imitation se porte sur des actes qui ont réussi et que le sujet a vu réussir par des personnes en qui il a confiance et qui ont autorité sur lui.

L'élément social se trouve dans le prestige de la personne qui fait l'acte ordonné, autorisé, prouvé par rapport à l'individu imitateur. L'imitation se fonde donc sur l'Autre signifiant.

Edward Hall explique en outre qu' "On ne peut pas < enseigner > la culture comme on enseigne le langage. » et ce faisant il se centre sur les aspects hors conscience de la culture et de la communication. En s'appuyant sur des distinctions préalables séparant «culture patente» et «culture latente» (Ralph Linton, 1967) ou encore « culture explicite » (les lois) et « implicite » (ce que l'homme considère comme inné, qui se développe en marge de la conscience (Clyde Kluckhonhn, 1964), E. Hall propose une catégorisation tripartite distinguant le formel, l'informel et le technique.

Cette distinction présente l'intérêt, pour la question qui nous occupe, d'être reliée à 
une identification parallèle de modalités de transmission particulières.

Selon cet auteur la connaissance formelle est transmise par injonction et par remontrances. Les schémas formels s'apprennent le plus souvent lorsqu'une faute commise se voit corrigée par quelqu'un. Les détails de la connaissance formelle sont bipolaires : oui/non, bien/mal. La connaissance informelle s'apprend par imitation d'un modèle. Cette transmission des connaissances informelles se fait le plus souvent de façon inconsciente aussi bien pour les apprenants que pour les modèles. La connaissance technique se transmet habituellement en termes explicites d'un professeur à un élève. Elle est souvent précédée d'une analyse logique et s'exprime en une forme générale cohérente. La transmission technique se fait donc dans le cadre d'un enseignement réfléchi à l'avance et structuré.

David le Breton (1992), émet quelques réserves quant aux travaux de Edward Hall, en particulier par rapport au fait que ce dernier méconnaît les différences groupales pour ne s'attacher qu'à des ensembles comme les "nations ", dont l'unité culturelle est douteuse. Cependant il souligne que l'apprentissage de "l'étiquette corporelle", c'est-à-dire le mode d'expression corporelle adapté à une situation sociale donnée, ne relève pas du même mode de transmission que celui des techniques corporelles, mais d'une éducation informelle, impalpable dans laquelle le mimétisme de l'acteur et les identifications jouent un rôle important. Cela confirme l'analyse du niveau informel » de Edward Hall, en le reliant à la notion d'étiquette corporelle.

A partir de cette catégorisation tripartite, nous allons essayer d'identifier ce qui, dans les usages du corps spécifiques des personnes en fauteuil roulant, est transmis, sous quelle forme, et par qui.

\section{L'Enseignement}

13 Les techniques corporelles spécifiques au public en fauteuil roulant sont transmises le plus souvent sous la forme d'un enseignement auquel participent les différents professionnels de la rééducation, médecins, kinésithérapeutes, ergothérapeutes, professeurs d'E.P.S. : on enseigne le "transfert", on enseigne comment descendre quelques marches d'escalier sur deux roues, on enseigne les techniques de soins corporels spécifiques et des techniques sportives spécifiques. Des personnes handicapées ayant l'expérience de ces techniques peuvent également participer à cet « enseignement » de techniques.

Des savoirs relatifs au corps handicapé et au fauteuil roulant sont également transmis sous la forme d'un enseignement plus ou moins structuré qui émane des professionnels de la rééducation: des savoirs médicaux le plus souvent, portant en particulier sur les complications secondaires possibles de l'atteinte médullaire, et les préventions et traitements de celles-ci.

\section{L'Education}

14 Les attitudes corporelles et l'habitus corporel ne sont par contre jamais enseignés au sens ici donné à ce terme. L'apprentissage de ceux-ci se fait dans le cadre d'une éducation formelle ou d'une transmission plus diffuse, processus qui se mettent en place clans les interactions entre personnes handicapées. Le valide, en tant que modèle corporel devenu caduc pour celui qui va vivre en fauteuil, ne semble plus pouvoir jouer ici de rôle éducatif. C'est dans le cadre des discussions, des rencontres 
entre pairs que se transmettent et que s'acquièrent des usages du corps propres au groupe, en partie à l'insu des acteurs. Mais d'autres savoirs sur le corps que les savoirs médicaux, plus pratiques ceux-là, transitent également par les pairs sous la forme d'une éducation formelle ou informelle.

Cette transmission d'une certaine culture corporelle qui échappe ici à l'enseignement classique mérite une exploration plus approfondie car la culture corporelle de ce groupe, aussi spécifique soit elle, n'en est pas moins fondamentalement articulée sur celle des « valides».

\subsection{L'éducation par «les générations adultes »}

15 Abordons le sujet de l'éducation par "les générations adultes sur celles qui ne sont pas encore mûres pour la vie sociale.»: hors de l'enseignement. Selon Emile Durkheim (1922), « Pour qu'il y ait éducation, il faut qu'il y ait en présence une génération d'adultes et une génération de jeunes, et une action exercée par les premiers sur les seconds. »(p. 47). Il précise encore sa définition de l'éducation en disant:

"L'éducation est l'action exercée par les générations adultes sur celles qui ne sont pas encore mûres pour la vie sociale. Elle a pour objet de susciter et de développer chez l'enfant un certain nombre d'états physiques, intellectuels et moraux que réclament de lui et la société politique dans son ensemble et le milieu spécial auquel il est particulièrement destiné.» (p. 51).

16 La notion d'adulte de E. Durkheim prend ici une forme particulière : c'est « l'adulte du fauteuil», "l'ancien», celui que l'expérience de la vie en fauteuil autorise à se transformer en «éducateur " pour ceux «qui ne sont pas encore mûrs pour la vie sociale en fauteuil », ceux qui, quel que soit leur âge, naissent à cette "vie assise » (les «novices», les «nouveaux»). Ceux qui ne peuvent plus courir et qui ne savent pas encore « rouler».

Cette éducation s'actualise soit dans le cadre d'activités organisées, comme les activités sportives par exemple, ou bien dans le cadre d'espaces plus informels de rencontre, que ce soit les couloirs, le café ou tout autre contexte dans lequel des anciens et des nouveaux se retrouvent.

\section{Culture spécifique et ouverture aux autres : une éducation à la déstigmatisation}

17 Dans cette éducation entre "anciens » et "nouveaux», on peut différencier des axes comme: le formel et l'informel, mais on peut aussi distinguer ce qui vise à une éducation corporelle pour un fonctionnement dans le groupe de pairs ou pour un fonctionnement avec les « valides ». C'est-à-dire ce qui vise à un renforcement de l'identité groupale (fonction assertive) et ce qui vise à une ouverture vers l'extérieur (fonction intégrative).

Il y a, par exemple, des discours formels, entre pairs, qui portent sur le corps et ses spécificités et qui transmettent aux nouveaux tout un tas de réponses à des questions qu'ils se posent: on parle des contractures, des soins, des règles pour les filles, des problèmes de miction, de la sexualité, de la paternité ou de la maternité... Il n'est pas rare d'ailleurs que ces discours s'opposent aux discours médicaux produits par les professionnels. 
Mais il y a aussi des discours formels qui sont des discours éducatifs (des " anciens " vers les « nouveaux ») portant sur les attitudes à avoir avec les « valides ». En effet, ce groupe semble posséder un certain nombre de savoirs spécifiques, relatifs à sa situation corporelle particulière, qui sont posés, de façon formelle, comme " internes », c'est-à-dire ne devant pas dépasser le groupe de pairs et le groupe des professionnels. Les "nouveaux » sont éduqués, par injonctions, à cette ligne de conduite, dont la transgression est considérée par le groupe comme préjudiciable à l'intégration sociale. Cette éducation propose, par exemple, d'éviter ou de limiter l'information des valides sur les aspects de soins particuliers du corps et de prise en charge de celui-ci qu'impose le handicap.

L'éducation informelle reproduit cette double perspective en proposant des modèles différenciés d'attitudes corporelles en fonction du contexte social. Dans le rapport avec les valides, elle ne propose pas de jouer sur la rétention d'information comme précédemment, mais sur la mise en scène du corps.

Un certain nombre d'attitudes corporelles observées chez les personnes en fauteuil apparaissent comme "calquées » sur le code de communication des valides. C'est en particulier le cas de différentes attitudes corporelles des sujets assis et impliquant les jambes : on observe par exemple que les sujets «se croisent les jambes", ou bien « se mettent un pied en appui sur une sangle », comme on mettrait un pied sur le barreau de la chaise. Ces attitudes corporelles, principalement observables dans les interactions handicapés-valides, peuvent être interprétées comme des stratégies de communication et de conjuration de la gêne provoquée chez les valides par un corps «hors-normes» et par le fauteuil roulant. Elles sont à comprendre comme une modalité de ce que David Le Breton appelle «l'effacement ritualisé du corps» (1984). La personne en fauteuil par l'adoption de ces attitudes tente en effet de répondre aux attentes corporelles des interactions ordinaires, pour « renvoyer en miroir » au valide une présentation $\mathrm{du}$ corps socialement codée et ritualisée qui participe de l'effacement de ce corps particulier, et donc de l'effacement du handicap.

Ces postures, cette gestuelle, "artificielles » dans le sens où elles nécessitent une manipulation des parties immobiles du corps, sont des recours au code corporel commun. Cette mise en scène contribue à l'effacement du handicap par la construction d'une image qui puisse «faire miroir» pour le valide en tentant de «faire oublier » le fauteuil. Mais plus encore, l'analyse de ces postures montre qu'elles renvoient le plus souvent à la symbolique de la "détente", de la "décontraction ", proposant ainsi, au-delà de l'effacement du corps, l'image positive d'un sujet «à l'aise » clans l'interaction sociale. Elles permettent alors «l'effacement ritualisé du handicap »et participent d'une logique de déstigmatisation (Marcellini, 1991). 


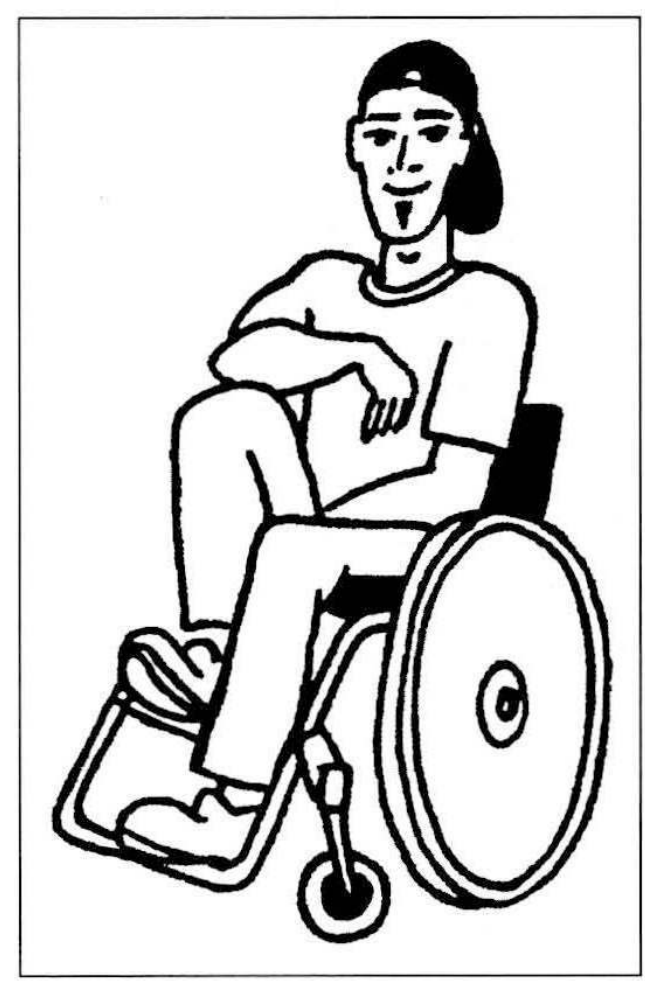

LES ATtitudes CORPORELLes AU REPos. Posture renvoyant à la symbolique de la détente, de la décontraction. Dessin de Jean-Pierre Thérond.

18 Erving Goffman (1981) avait déjà souligné la dimension de la communication, voulue ou non, mais toujours contenue dans «l'apparence physique et des actes personnels tels que l'habillement, le maintien, les mouvements et les attitudes, l'intensité de la voix, les gestes comme le salut ou les signes de la main, l'ornementation du visage et l'expression émotionnelle en général » (p. 267). Et il ajoutait que «la compréhension d'un dialecte corporel commun est une des raisons d'appeler un ensemble d'individus une société»(p. 269). La logique « d'effacement ritualisé du handicap » repérée ici confirme la logique intégrative du groupe qui éduque les "nouveaux» à une réappropriation du dialecte corporel signifiant pour les « valides » qui les entourent.

Il faut, à ce propos, souligner notre étonnement (de valide) lorsque des personnes paralysées d'une seule jambe et ayant la possibilité de se déplacer « debout » avec des béquilles décident de «se mettre en fauteuil ». Au-delà de la justification technique d'un tel choix (traumatisme des épaules, fatigue), nous pouvons ici penser que la déambulation avec béquilles, et l'obligation de se tenir debout à la force des bras ne permettent pas une mise en scène du corps se rapprochant de l'« ordinaire ». Le fait de boiter, de se déhancher pour marcher choque le regard par le «défaut " par rapport à la marche ordinaire et le miroir est brisé. Etre en fauteuil au contraire permet de maintenir le buste droit, de développer une technique de déplacement efficace, et surtout d'offrir l'image d'un corps certes « assis », mais contrôlé, dompté et socialisé. La libération des mains permet en outre l'adoption de toute cette gestuelle facilitant une mise en scène du corps et une présentation de soi compatibles avec le système d'attente de l'environnement culturel. 


\section{Peut-on enseigner une culture corporelle?}

19 Il apparaît donc au travers de l'étude de ce groupe particulier, qu'au-delà de l'enseignement, qui couvre une part de la transmission de la culture corporelle, c'est l'éducation dans ses modalités formelles et informelles qui conduit le «nouveau » dans le passage d'une culture «debout » à une culture « en fauteuil ».

Plus encore, la position particulière de ce groupe, sous-groupe de notre société, nous fournit l'illustration d'une éducation corporelle préparant les sujets non seulement à une autonomie optimale pour chacun et au bien-être corporel dans le groupe, mais aussi à la gestion des relations entre une culture corporelle spécifique et la culture dominante : une sorte d'éducation à l'intégration au sens le plus complet du terme.

Ce groupe, en tant que minorité, fait preuve ici d'une logique éducative qui explique peut-être, en partie, son passage rapide de la ségrégation stigmatisante à une participation sociale négociée qui évite l'écueil de l'assimilation pure et simple.

Pour conclure sur la question «Comment peut-on enseigner une culture corporelle?", l'analyse menée ici semble indiquer que si l'on peut enseigner une part de culture corporelle, les techniques, tout ce qui relève du formel et de l'informel échappe à l'enseignement structuré, rationalisé.

Mais il faut nuancer cette conclusion. En effet, les modalités de transmission observées dans notre étude, l'ont été ici et maintenant, et elles sont susceptibles d'évolution. Il faut savoir, par exemple, que des groupes de personnes handicapées travaillent en ce moment même à l'élaboration d'une pratique de ce qu'ils nomment la «pair-émulation $»^{1}$. Cela renvoie à une volonté d'institutionnalisation et de structuration de ce que nous avons identifié comme le formel et l'informel de cette culture : accueillir les «nouveaux » pour les former à la nouvelle vie qui les attend. Demain, l'éducation par les pairs pourra donc peut être, en partie, prendre la forme d'un enseignement? En effet, on comprend que l'étude et l'analyse du formel et de l'informel d'une culture donnée permet un changement de statut des contenus formels et informels, qui peuvent devenir alors « techniques».

Il semble alors que l'on puisse effectivement « enseigner une culture corporelle ", à condition que les contenus culturels, par le biais d'une analyse deviennent « techniques ». C'est-à-dire que l'on parvienne à les conscientiser et à les rationaliser. Restent donc le non-rationnel et l'inconscient de la culture...

\section{BIBLIOGRAPHIE}

COLLOQUE Vie AUTONOME - 1997, Organisé par le Centre ProPaRa et le G.I.H.P. LanguedocRoussillon, les 14 \& 15 mars 1997. (Document photocopié).

DURKHEIM E., Education et sociologie, Paris, Éd. Quadrige, 4e Éd., 1922, Ré-éd. : 1993.

GOFFMAN E., « Engagement », in La nouvelle communication, Paris, Seuil, p. 267-278, 1981. 
HALL E.T., The silent langage. Garden city, St. : New York, U.S.A.. Publisher : Doubleday \& Company Inc., 1959. (Edition française : Le langage silencieux, Paris, Seuil, 1984).

KLUCKHONHN C, Initiation à l'anthropologie, Bruxelles, Belgique, Éd. Ch. Dessart, 1964.

LE BRETON D., La sociologie du corps. Paris, PUF, 1992, 2e Éd. : 1994.

LE BRETON D., « La symbolique corporelle », in Ethnologie française. Vol. 15-Nº1, p. 73-78, 1985.

LE BRETON D., «L'effacement ritualisé du corps », in Cahiers internationaux de sociologie, Vol. 77, p. 273-281, 1984.

LE BRETON D., "Corps et symbolique sociale ", in Cahiers internationaux de sociologie. Vol. 73. p. 223-232, 1982.

LINTON R., Le fondement culturel de la personnalité. Paris Dunod, 1967.

MARCELLINI A., Sport, Stigmate et intégration sociale: contribution à l'étude des stratégies de déstigmatisation- Thèse de doctorat. Montpellier, Éd. Université Montpellier I, 1991.

MAUSS M., Manuel d'ethnographie. Paris, Payot, 1947. 3e Éd. : 1989.

MAUSS M., Sociologie et anthropologie. Paris, PUF. 1950, 7e Éd. : 1997.

MURPHY R., The body solent- A journey into paralysis, Henry Holt \& Company publishers, New York City, U.S.A., 1987 (Edition française : Vivre à corps perdu, Paris, Plon, 1990).

PICARD D., " Approche ethnopsychologique du corps », in Cahiers de sociologie économique et culturelle. $\mathrm{N}^{\circ} 3$. p. 23-33. 1985.

\section{NOTES}

1. Ce terme est la traduction de l'expression «Peer Counseling », une des bases de réflexion du European Network for Independant Living (Mouvement pour la Vie Autonome). La Pair-émulation est définie comme « la transmission de l'expérience par les personnes handicapées autonomes, pour les personnes handicapées en recherche de plus d'autonomie, et avec le but de renforcer la conscience des personnes handicapées sur leur possibilités, leurs droits et leurs devoirs". (Colloque « Vie Autonome », 1997)

\section{RÉSUMÉS}

Comment s'opère le passage d'une «culture debout» à une "culture en fauteuil» ? Cette étude illustre parfaitement ce qui peut s'enseigner (les techniques du corps formalisées), et ce qui ne peut l'être (ce qui appartient à l'irrationnel et à l'inconscient).

How does the transition from "standing" culture to a "sitting" (in a wheel - chair) culture work? This study illustrates perfectly what can he taught (the body techniques expressed in words), and what cannot (what belongs to the domain of the irrational and the subconscious). 
INDEX

Keywords : culture, handicap, particularité, specificity body technique, technique du corps

AUTEUR

ANNE MARCELLINI

UFR-STAPS, Université de Montpellier 1 